\title{
On the Importance of Electronic Symmetry for Triplet State Delocalization
}

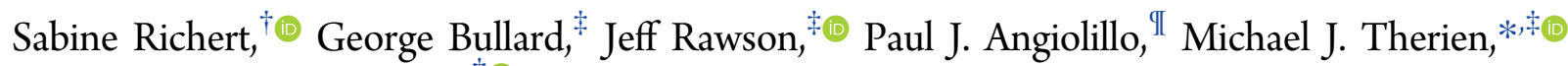 \\ and Christiane R. Timmel*, ${ }^{*}$ (i) \\ ${ }^{\dagger}$ Centre for Advanced Electron Spin Resonance (CAESR), University of Oxford, South Parks Road, Oxford OX1 3QR, United \\ Kingdom \\ ${ }^{\ddagger}$ Department of Chemistry, Duke University, French Family Science Center, 124 Science Drive, Durham, North Carolina 27708, \\ United States \\ IIDepartment of Physics, Saint Joseph’s University, 5600 City Avenue, Philadelphia, Pennsylvania 19131, United States
}

\section{Supporting Information}

\begin{abstract}
The influence of electronic symmetry on triplet state delocalization in linear zinc porphyrin oligomers is explored by electron paramagnetic resonance techniques. Using a combination of transient continuous wave and pulse electron nuclear double resonance spectroscopies, it is demonstrated experimentally that complete triplet state delocalization requires the chemical equivalence of all porphyrin units. These results are supported by density functional theory calculations, showing uneven delocalization in a porphyrin dimer in which a terminal ethynyl group renders the two porphyrin units inequivalent. When the conjugation length of the molecule is further increased upon addition of a second terminal ethynyl group that restores the symmetry of the system, the triplet state is again found to be completely delocalized. The observations suggest that electronic symmetry is of greater importance for triplet state delocalization than other frequently invoked factors such as conformational rigidity or fundamental length-scale limitations.
\end{abstract}

\nowledge of the factors governing excited state delocaliza- 1 ion is of paramount importance for numerous applications in the fields of spintronics, molecular electronics, and photovoltaics. ${ }^{1-3}$ In particular, the triplet photoexcited state has gained prominence as an entity to expand the region of the electromagnetic spectrum that can be used for solar energy conversion. In this context, it has fueled many opportunities in solar harvesting, photocatalysis, or bioimaging through photon upconversion mediated by triplet-triplet annihilation. ${ }^{4-7}$ To develop and improve further applications in this active area of research, understanding the fundamental photophysics of triplet generation, triplet lifetime control, and delocalization thus becomes increasingly relevant.

meso-to-meso Ethyne-bridged porphyrin motifs evince highly delocalized $S_{1}$ states resulting from strongly coupled electronic communication between porphyrin units. ${ }^{8,9}$ Recent studies probing charged excitations (polaron states) of these porphyrin oligomers demonstrated unprecedented delocalization lengths of greater than $7.5 \mathrm{~nm} .{ }^{10,11}$ These systems also possess triplet states resulting from intersystem crossing that have been well characterized optically and by continuous wave $(\mathrm{cw})$ electron paramagnetic resonance (EPR). ${ }^{12-15}$

In previous studies on triplet states of ethyne-linked linear porphyrin oligomers, EPR and transient absorption data were interpreted in terms of triplet excitations that were more spatially confined than the corresponding singlet excited states. ${ }^{12,14,15}$ These results were further supported by timeresolved microwave conductivity measurements on butadiynelinked porphyrins. ${ }^{16,17}$

Recent studies exploiting transient EPR and electron nuclear double resonance (ENDOR) techniques have more richly characterized photoexcited triplet states in butadiyne-linked linear and cyclic porphyrin oligomers. ${ }^{18-20}$ These studies showed complete triplet state delocalization for a meso-to-meso butadiyne-bridged porphyrin dimer, while investigation of longer, linear butadiyne-linked porphyrin oligomers comprising three to six porphyrin units demonstrated an uneven spin density distribution with the majority of the spin density located on the central porphyrin units of the structures. Importantly, complete delocalization over all zinc porphyrin units was observed in a corresponding template-bound sixmembered nanoring. ${ }^{19}$ It is the latter result that seems to hold the key to a better understanding of the factors driving triplet state delocalization: the features that distinguish the cyclic sixmembered zinc porphyrin nanoring with respect to the linear porphyrin structures are (i) the bent, circular, rigid geometry of the porphyrin $\pi$-system and (ii) the chemical equivalence of all porphyrin units resulting in a cylindrically symmetric molecular electronic system.

The influence of bending of the porphyrin $\pi$-system on triplet state delocalization has been studied before by coordinating butadiyne-linked linear porphyrin oligomers to radial molecular templates. ${ }^{19}$ In the presence of the hexagonal template, the porphyrin chains are forced to adopt a bent and rigid circular arc geometry. The EPR results evince smaller zero-field splitting $D$-values, but the proton ENDOR couplings suggest that the triplet state electron density distribution

Received: February 3, 2017

Published: March 29, 2017 


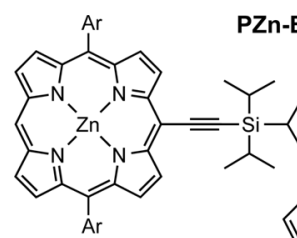

$\mathrm{PZn}_{2}$

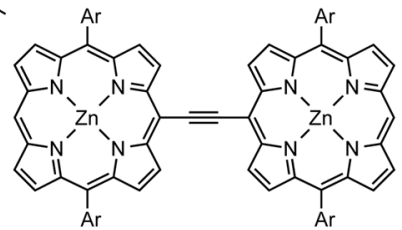

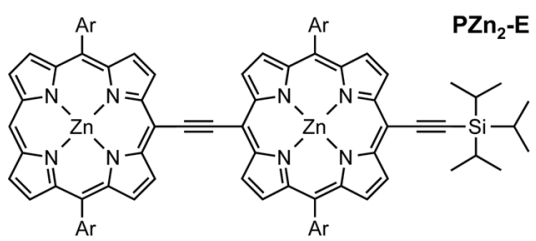

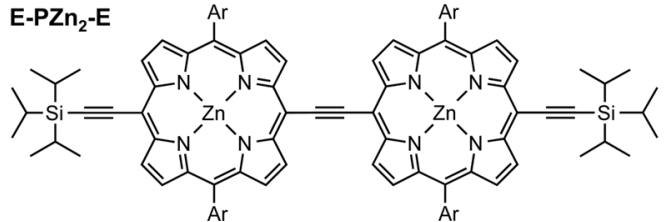

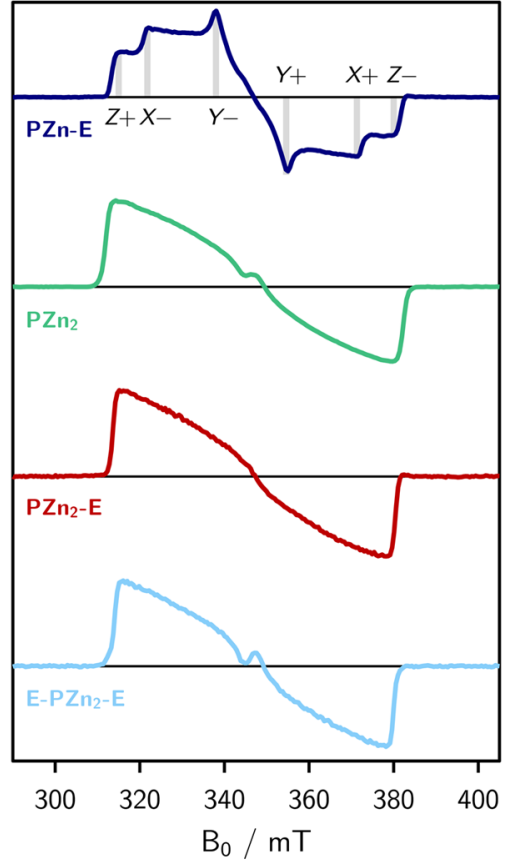

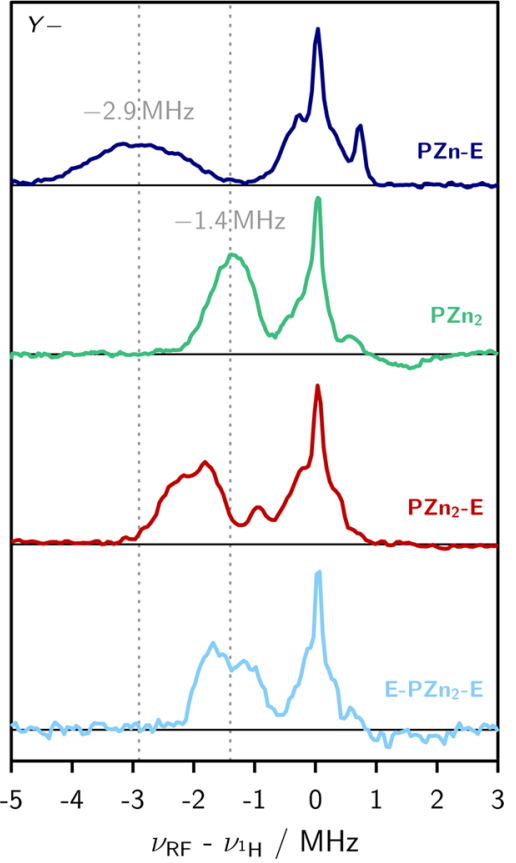

Figure 1. Chemical structures (left), comparison of the transient cw EPR spectra (center) and proton Mims ENDOR spectra recorded at a field position corresponding to the $Y$ - orientation (right). The EPR and ENDOR data were acquired in frozen MTHF at $20 \mathrm{~K}$. Pulse lengths of $16 \mathrm{~ns}$ were applied in the ENDOR experiments and three spectra with $\tau$ values of 120, 180, and 240 ns were averaged in all cases to yield the depicted spectra where the effect of the blind spots is compensated (cf. SI). The canonical orientations are indicated for PZn-E. The gray dotted lines indicate the magnitude of the hyperfine coupling for PZn-E and half of this value, respectively. See SI for further details.

remains unperturbed and localized, mirroring the data obtained for the linear untemplated analogs. ${ }^{19,20}$

Collectively, these findings suggest that the equivalence of the porphyrin units reflected in the molecule's overall symmetry might be the decisive factor controlling the spatial extent of the triplet excitation.

To confirm this statement, the importance of electronic symmetry for triplet state delocalization is investigated on a series of ethyne-linked porphyrin oligomers. In density functional theory (DFT) calculations of similar porphyrin systems, it is observed that a considerable amount of spin density is located on the triple bond linker between the porphyrin units as well as the terminal triple bonds. ${ }^{18,19}$ In addition, electronic control of the photoexcited triplet state via meso-ethyne elaboration of a (porphinato) $\mathrm{Zn}$ (II) core has also been shown experimentally; increasing the number of meso-ynyl units induces an electronic spin density redistribution from an oblate to a more prolate one with a concomitant increase in the spatial extent of the triplet wavefunction. ${ }^{21}$ Considering these results, a series of three meso-to-meso ethyne-bridged bis[(porphinato)zinc] chromophores ( $\mathbf{P Z n _ { 2 }}$ structures) that differ with respect to the extent of terminal meso-carbon conjugation were examined to explore the influence of electronic symmetry on triplet state delocalization.

The dimer structures are referred to as $\mathbf{P Z n}_{2}, \mathbf{P Z n _ { 2 }}-\mathbf{E}$, and E$\mathbf{P Z n}_{2}-\mathbf{E}$ and are shown in Figure 1 (left). According to our hypothesis above, it should be possible to control the triplet state delocalization in these structures via their molecular symmetry, i.e., via the presence or absence of the terminal ethynyl groups. As further benchmarks, monomers with one or two terminal triple bonds, PZn-E and E-PZn-E, and the trimer without end groups, $\mathbf{P Z n _ { 3 }}$, were also studied. The results for $\mathbf{E}$ PZn-E and $\mathbf{P Z n}_{3}$ are presented in the SI.
The EPR samples of the investigated compounds were prepared at a concentration of $\sim 0.2 \mathrm{mM}$ in 2-methyltetrahydrofuran (MTHF) and the measurements were performed in frozen solution at $20 \mathrm{~K}$ (details are given in the SI). As in previous work, the hyperfine coupling constants, measured by pulse ENDOR, are used to determine the extent of triplet state delocalization because they directly reflect the electronic spin density of the system.

Information about delocalization could in principle also be obtained from the zero-field splitting (ZFS) parameter $D$, which is related to the overall width of the triplet state EPR spectrum. However, the $D$-value does depend not only on the average delocalization length but also on the shape of the spin density distribution. If the latter changes, for example, from an oblate to a gradually more prolate one within a series of molecules, the $D$-values are not directly comparable and accurate information on the orientation of the ZFS tensor is needed for interpretation ${ }^{18}$ (see SI).

To obtain the triplet state EPR spectra and determine the ZFS parameters $D$ and $E$, transient cw EPR spectroscopy was applied. For the monomer, $D>0$ as determined by magnetophotoselection experiments. ${ }^{18}$ In this study, we chose the convention $|Z| \geq|X| \geq|Y|$, implying $E<0$ for the monomer. The $Y$ axis of the ZFS tensor corresponds to the axis along the aryl groups. As conjugation length increases in meso-to-meso ethyne- and butadiyne-linked (porphinato)zinc oligomers, $\mathrm{cw}$ EPR line shape analysis signaled a reorientation of the ZFS tensor, eventually yielding a prolate spin distribution with $D<0$ and $E>0,{ }^{12-15}$ a finding corroborated in butadiyne-linked porphyrins by magnetophotoselection experiments and analysis of the ${ }^{1} \mathrm{H}$ hyperfine couplings. ${ }^{18}$ Whereas the $Z$ and $X$ axes are interchanged upon such a reorientation of the ZFS tensor (i.e., transition from an oblate to a prolate spin density distribution), 
the $Y$ axis still points along the aryl groups in the longer oligomers (see SI for details). ${ }^{12-15}$

The experimental triplet state spectra for $\mathbf{P Z n}-\mathbf{E}, \mathbf{P Z n _ { 2 }}$, $\mathbf{P Z n}_{2}-\mathbf{E}$, and $\mathbf{E}-\mathbf{P Z n _ { 2 }}-\mathbf{E}$ are shown in Figure 1 (center). No significant change in the width of the spectrum and therefore the $D$-value is observed between the monomer and dimers. In agreement with earlier work ${ }^{12-15,18}$ and DFT calculations (see SI), this can be attributed to increased delocalization in combination with a change in the spin density distribution (i.e., the orientation of the ZFS tensor). As the conjugation length increases from $\mathbf{P Z n _ { 2 }}$ to $\mathbf{P Z n _ { 2 }}-\mathbf{E}$, a slight decrease in the width of the triplet spectrum is observed (see SI). Further augmenting the conjugation length from $\mathbf{P Z n _ { 2 }}-\mathbf{E}$ to E-PZn $\mathbf{n}_{2}-\mathbf{E}$ results in no further significant reduction in the $D$-value. A more quantitative analysis and interpretation of the spectra including simulations to determine $D$ and $E$, and relative populations of the triplet sublevels, are presented in the SI.

Since previous investigations revealed that more reliable conclusions on triplet state delocalization can be drawn from ENDOR results, Mims ${ }^{1} \mathrm{H}$ ENDOR spectra were recorded for all compounds at magnetic field positions corresponding to the $Y$ - canonical orientation, where the largest hyperfine couplings were observed. The spectra are shown in Figure 1 (right). The magnitudes of the largest $\beta$-proton hyperfine couplings in the $Y$ - orientation, which will be compared in the following, correspond to a projection of the hyperfine coupling tensors shown in light blue in Figure 2 (right) onto the $Y$ axis of the respective ZFS tensor. ${ }^{22}$

A broad peak centered at $-2.9 \mathrm{MHz}$ is observed in the ENDOR spectrum of PZn-E. The corresponding coupling is approximately halved in the case of $\mathbf{P Z \mathbf { n } _ { 2 }}$, indicating complete delocalization over two porphyrin units. The width of the ENDOR peak is slightly larger in the case of PZn-E, presumably due to a larger heterogeneity of the individual couplings as a consequence of the slight symmetry distortion induced by the terminal triple bond. A similar largest coupling as in $\mathbf{P Z n}_{2}$, of about $-1.4 \mathrm{MHz}$, is measured in the symmetric dimer with two terminal triple bonds, E-PZn $\mathbf{n}_{2}-\mathbf{E}$, whereas larger couplings are present in the spectrum of the asymmetric dimer, $\mathrm{PZn}_{2}-\mathrm{E}^{23}$

These data clearly demonstrate that delocalization is not governed only by the length of the porphyrin $\pi$-system. When

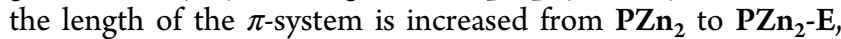
the magnitude of the largest hyperfine coupling constant increases, which can be interpreted as arising from an uneven

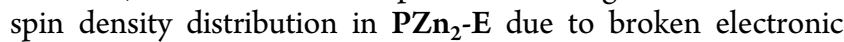
symmetry (i.e., inequivalence of the porphyrin units). However, upon further increase of the conjugation length from $\mathbf{P Z n _ { 2 }}-\mathbf{E}$ to $\mathbf{E}-\mathrm{PZn}_{2}-\mathbf{E}$, complete delocalization is evident as the electronic symmetry is restored. When a third porphyrin unit is added to the system $\left(\mathbf{P Z n}_{3}\right.$, see SI), the terminal porphyrins of the array are chemically inequivalent with respect to the central porphyrin unit. ${ }^{12-15}$ Therefore, uneven delocalization is expected, implying a slight increase in the largest hyperfine coupling constant with respect to the symmetric dimers. ENDOR results for the trimer, $\mathbf{P Z n}_{3}$, are shown in the SI and confirm this interpretation. A largest hyperfine coupling in the $Y$-direction of $-2 \mathrm{MHz}$ was measured, indicating an increased spin density on the central porphyrin unit. Consequently, in agreement with the results of previous studies, ${ }^{12,14,15,18,19}$ uneven triplet spin delocalization should in general be expected for any linear systems with more than two units, since the inequivalence of the "inner" and "outer" units persists.
To support this tentative explanation, DFT calculations of the hyperfine coupling constants and triplet spin density distributions were carried out. The aryl side groups were replaced by phenyl groups, and any triisopropylsilyl (TIPS) protecting groups, if present in the structures, were replaced by protons to simplify the calculations (details provided in the SI).

Figure 2 illustrates the calculated proton hyperfine coupling tensors (right) and triplet spin densities (left) in the molecular

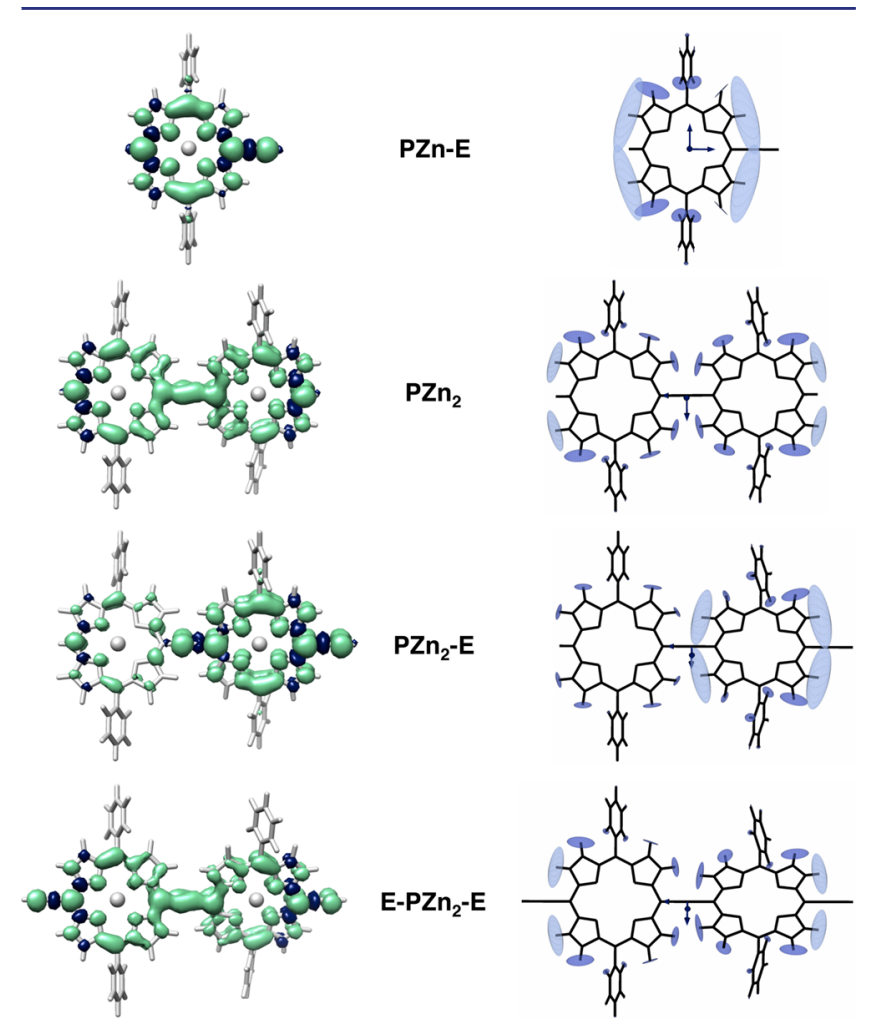

Figure 2. Calculated triplet spin density distributions (left) and visualization of the calculated hyperfine coupling tensors (right) for the four investigated structures.

frames of PZn-E, $\mathbf{P Z n _ { 2 }}, \mathbf{P Z n _ { 2 }}-\mathbf{E}$, and E-PZn $\mathbf{n}_{2}-\mathbf{E}$. The tensors corresponding to the largest coupling in the $Y$-direction, compared among the different oligomers, are highlighted in the figure in light blue, and provide information on the extent of delocalization. Due to the presence of a terminal meso-proton

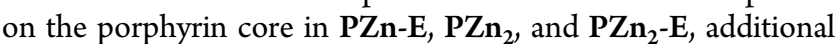
peaks should be observable in the ENDOR spectra of these compounds. These comparatively large couplings are omitted in the figure for clarity (see SI for details).

The results from DFT calculations predict an uneven spin

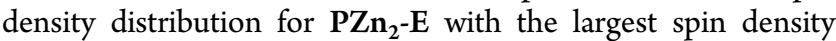
found on the porphyrin unit with the additional ethynyl end group, whereas symmetric delocalization is predicted for $\mathbf{P Z \mathbf { n } _ { 2 }}$ and $\mathbf{E}-\mathbf{P Z n} \mathbf{n}_{\mathbf{2}}-\mathbf{E}$. The relative changes in the magnitudes of the hyperfine coupling constants coincide well with the experimentally observed trends and confirm the interpretation of the results presented above. The calculated magnitudes and the resulting assignment of the experimental ENDOR peaks to the individual protons of the structures are given in the SI.

In conclusion, the influence of electronic symmetry on triplet state delocalization was explored: both experimentally and theoretically, the triplet state is found to be unevenly delocalized in $\mathbf{P Z n _ { 2 }}-\mathbf{E}$ but completely delocalized in $\mathbf{E}-\mathbf{P Z n _ { 2 }}-$ 
$\mathrm{E}$, where the $\pi$-conjugation length is increased as compared to $\mathbf{P Z n} \mathbf{n}_{\mathbf{2}}$-E. These results indicate that the observed limitation of the extent of triplet state delocalization to about two porphyrin units in linear porphyrin oligomers is not fundamental in nature, i.e., the triplet state is not automatically localized over only a small part of the molecule as often assumed. The extent of triplet state delocalization appears related to the chemical inequivalence reflected at the antipodal ends of these conjugated $\mathbf{P Z \mathbf { n } _ { 2 }}$ supermolecules.

In the symmetric molecules, $\mathbf{P Z n _ { 2 }}$ and $\mathbf{E}-\mathbf{P Z n _ { 2 }}-\mathbf{E}$, both porphyrin units are chemically equivalent, whereas in $\mathbf{P Z n _ { 2 }}-\mathbf{E}$ the electronic symmetry is broken, leading to an uneven spin density distribution. The results of this study suggest that complete delocalization can only be observed up to two porphyrin units in linear oligomers: beginning with the trimer, the "inner" and "outer" porphyrin units of the chain will always be inequivalent, resulting in an uneven spin density distribution as observed previously. The findings are also in agreement with the observation of complete delocalization in a symmetrical cyclic porphyrin hexamer, ${ }^{19}$ where all units are chemically equivalent.

This work demonstrates the importance of the chemical equivalence of the individual monomeric units for triplet state delocalization in oligomeric porphyrin assemblies, which confirms the tentative conclusions of an earlier study. ${ }^{19}$ Based on the results presented here, it is speculated that electronic symmetry is of greater importance for triplet state delocalization than other frequently invoked factors such as conformational rigidity or fundamental length-scale limitations.

\section{ASSOCIATED CONTENT}

\section{S Supporting Information}

The Supporting Information is available free of charge on the ACS Publications website at DOI: 10.1021/jacs.7b01204.

Compound characterization, experimental methods, complementary EPR and ENDOR data and simulations, DFT calculations (PDF)

\section{AUTHOR INFORMATION}

\section{Corresponding Authors}

*michael.therien@duke.edu

*christiane.timmel@chem.ox.ac.uk

\section{ORCID 1}

Sabine Richert: 0000-0002-2990-974X

Jeff Rawson: 0000-0002-8431-0772

Michael J. Therien: 0000-0003-4876-0036

Christiane R. Timmel: 0000-0003-1828-7700

\section{Notes}

The authors declare no competing financial interest.

\section{ACKNOWLEDGMENTS}

Financial support from the EPSRC (EPL011972/1), the ERC (grant 320969), and the Division of Chemical Sciences, Geosciences, and Biosciences, Office of Basic Energy Sciences, of the U.S. Department of Energy through grant DESC0001517, is gratefully acknowledged. The authors acknowledge the use of the University of Oxford Advanced Research Computing (ARC) facility in carrying out this work (http://dx. doi.org/10.5281/zenodo.22558).

\section{REFERENCES}

(1) Wolf, S. A.; Awschalom, D. D.; Buhrman, R. A.; Daughton, J. M.; von Molnár, S.; Roukes, M. L.; Chtchelkanova, A. Y.; Treger, D. M. Science 2001, 294, 1488-1495.

(2) Ratner, M. Nat. Nanotechnol. 2013, 8, 378-381.

(3) Bakulin, A. A.; Rao, A.; Pavelyev, V. G.; van Loosdrecht, P. H. M.; Pshenichnikov, M. S.; Niedzialek, D.; Cornil, J.; Beljonne, D.; Friend, R. H. Science 2012, 335, 1340-1344.

(4) Parker, C. A.; Hatchard, C. G. Proc. Chem. Soc. 1962, 386-387.

(5) Singh-Rachford, T. N.; Castellano, F. N. Coord. Chem. Rev. 2010, $254,2560-2573$.

(6) Singh-Rachford, T. N.; Nayak, A.; Muro-Small, M. L.; Goeb, S.; Therien, M. J.; Castellano, F. N. J. Am. Chem. Soc. 2010, 132, 1420314211.

(7) Gray, V.; Dzebo, D.; Abrahamsson, M.; Albinsson, B.; MothPoulsen, K. Phys. Chem. Chem. Phys. 2014, 16, 10345-10352.

(8) Lin, V. S.-Y.; DiMagno, S. G.; Therien, M. J. Science 1994, 264, 1105-1111.

(9) Duncan, T. V.; Susumu, K.; Sinks, L. E.; Therien, M. J. J. Am. Chem. Soc. 2006, 128, 9000-9001.

(10) Susumu, K.; Frail, P. R.; Angiolillo, P. J.; Therien, M. J. J. Am. Chem. Soc. 2006, 128, 8380-8381.

(11) Rawson, J.; Angiolillo, P. J.; Therien, M. J. Proc. Natl. Acad. Sci. U. S. A. 2015, 112, 13779-13783.

(12) Angiolillo, P. J.; Lin, V. S.-Y.; Vanderkooi, J. M.; Therien, M. J. J. Am. Chem. Soc. 1995, 117, 12514-12527.

(13) Shediac, R.; Gray, M. H. B.; Uyeda, H. T.; Johnson, R. C.; Hupp, J. T.; Angiolillo, P. J.; Therien, M. J. J. Am. Chem. Soc. 2000, 122, 7017-7033.

(14) Angiolillo, P. J.; Susumu, K.; Uyeda, H. T.; Lin, V. S.-Y.; Shediac, R.; Therien, M. J. Synth. Met. 2001, 116, 247-253.

(15) Angiolillo, P. J.; Uyeda, H. T.; Duncan, T. V.; Therien, M. J. J. Phys. Chem. B 2004, 108, 11893-11903.

(16) Piet, J. J.; Taylor, P. N.; Anderson, H. L.; Osuka, A.; Warman, J. M. J. Am. Chem. Soc. 2000, 122, 1749-1757.

(17) Piet, J. J.; Taylor, P. N.; Wegewijs, B. R.; Anderson, H. L.; Osuka, A.; Warman, J. M. J. Phys. Chem. B 2001, 105, 97-104.

(18) Tait, C. E.; Neuhaus, P.; Anderson, H. L.; Timmel, C. R. J. Am. Chem. Soc. 2015, 137, 6670-6679.

(19) Tait, C. E.; Neuhaus, P.; Peeks, M. D.; Anderson, H. L.; Timmel, C. R. J. Am. Chem. Soc. 2015, 137, 8284-8293.

(20) Tait, C. E.; Neuhaus, P.; Peeks, M. D.; Anderson, H. L.; Timmel, C. R. Phys. Chem. Chem. Phys. 2016, 18, 5275-5280.

(21) Angiolillo, P. J.; Rawson, J.; Frail, P. R.; Therien, M. J. Chem. Commun. 2013, 49, 9722-9724.

(22) ENDOR spectra were also measured at the $X$ and $Z$ canonical orientations. The same trends were observed, supporting the conclusions drawn based on the spectra recorded at the $Y$ orientations. For reference, the ENDOR spectra for all compounds recorded at $X_{-}^{-}$, $Y-$, and $Z-$ are shown in the SI together with a detailed analysis and assignment of the hyperfine couplings to the individual protons.

(23) Although only one major coupling is expected in the $Y-$ ENDOR spectrum of E-PZn $\mathbf{n}_{2}-\mathbf{E}$, the corresponding peak at $-1.4 \mathrm{MHz}$ is found to have two local maxima. The two observed couplings likely reflect a greater degree of nuclear magnetic heterogeneity of its four sets of chemically inequivalent $\beta$-protons in $\mathbf{E}-\mathbf{P Z n}_{2}-\mathbf{E}$ relative to $\mathbf{P Z n _ { 2 }}$ in agreement with DFT calculations of the hyperfine coupling constants tabulated in the SI. 